\title{
Manufacturing intelligence and innovation for digital manufacturing and operational excellence
}

\author{
Chen-Fu Chien • Mitsuo Gen • Yongjiang Shi • \\ Chia-Yu Hsu
}

Published online: 21 March 2014

(C) Springer Science+Business Media New York 2014

Global manufacturing networks and supply chains are facing ongoing dynamic changes in the evolutionary business ecosystems. The introduction of new process technologies and the advances in manufacturing intelligence capabilities are having profound effects on the strategies, decisions, management, and operations involved in manufacturing (Chien et al. 2007; Leachman et al. 2007). While big data is accumulated due to the fully automation manufacturing facilities and logistics systems for business integration, various solutions and techniques are developed to extract useful information and derive effective intelligence to address new challenges such as cycle time reduction (Kuo et al. 2011; Chien et al. 2011), defect diagnosis (Hsu and Chien 2007; Liu and Chien 2013), demand forecast (Chien et al. 2010), equipment management (Chien et al. 2008; Hsu et al. 2012; Chien et al. 2013a), human capital (Chen and Chien 2011), and bio informatics (Lin and Chien 2009). In particular, manufacturing innovation and manufacturing intelligence technologies are developed to empower manufacturing excellence via soft computing, decision technologies, and evolutionary

\section{C.-F. Chien $(\varangle)$}

Department of Industrial Engineering and Engineering Management, National Tsing Hua University, Hsinchu 30013, Taiwan

e-mail: cfchien@mx.nthu.edu.tw

\section{Gen}

Fuzzy Logic System Institute, Fukuoka, Japan

e-mail: gen@ flsi.or.jp

\section{Y. Shi}

Institute for Manufacturing, Cambridge University, Cambridge, UK

e-mail: ys@eng.cam.ac.uk

\section{C.-Y. Hsu}

Department of Information Management, Yuan Ze University, Chungli 32003, Taiwan

e-mail: cyhsu@saturn.yzu.edu.tw algorithms to construct intelligent algorithms and solutions that can be embedded in various information systems for enterprise resources planning (ERP), advanced production system (APS), advanced process control and advanced equipment control (APC/AEC), manufacturing execution system (MES), engineering data analysis (EDA), and supply chain management (SCM) to enhance decision quality and management effectiveness (Chien et al. 2012; Chien et al. 2013b; Wu et al. 2012a; Wu et al. 2012b). By seamless integration of intelligence and decision technologies combined recent IT technology, manufacturing intelligence have been completely become the trend of. This special issue of the Journal of Intelligent Manufacturing (JIM) aims to address the critical needs for future factories, logistics, and global supply chain for Manufacturing Intelligence and Innovation for Digital Manufacturing and Operational Excellence.

This special issue is developed to exchange the foresights and disseminate the research results on Manufacturing Intelligence and Innovation for Digital Manufacturing and Operational Excellence. The special issue presents insightful, comprehensive, theoretical, practical, and real-world applications of advanced decision and intelligence technologies in manufacturing and logistics. Original, high quality papers of theoretical developments and empirical studies with scientific novelty and insights were invited from the scholars presented in the 2012 International Symposium on Semiconductor Manufacturing Intelligence (ISMI2012), January 68, 2012 at the Hsinchu, Taiwan, while other papers were directly submitted for review and publication. All the submitted manuscripts were carefully blind peer reviewed by at least two referees, in accordance with Journal of Intelligent Manufacturing procedure and finally fourteen papers were selected for this special issue.

The selected papers in this special issue are clustered into four interrelated groups to address the theme of this special 
issue from various perspectives. The first cluster addresses the important issues for manufacturing intelligence methodologies for multiobjective scheduling. In particular, "Multiobjective evolutionary algorithm for manufacturing scheduling problems: state-of-the-art survey" by Gen and Lin, addresses the design of multiobjective evolutionary algorithms (MOEAs) to solve a variety of scheduling problems including job shop scheduling (JSP), flexible JSP, automatic guided vehicle (AGV) dispatching in flexible manufacturing system (FMS), integrated process planning and scheduling. "Cooperative estimation of distribution algorithm: a novel approach for semiconductor final test scheduling problems" by $\mathrm{Hao}, \mathrm{Wu}$, Chien, and Gen proposes a novel cooperative estimation of distribution algorithm (CEDA) that incorporates a cooperative co-evolutionary paradigm to extend the model features and improve the evaluation lead-time of estimation of distribution algorithm (EDA), outperforming hybrid GAs for several semiconductor final test scheduling (SFTS) problems. "Hybrid Sampling Strategy-based Multiobjective evolutionary algorithm for process planning and scheduling problem" by Zhang, Gen, and Jo, develops a hybrid sampling strategy-based multiobjective evolutionary algorithm (HSS-MOEA) to solve process planning and scheduling problem.

The second cluster focuses on complex challenges in modern production facilities for operation effectiveness. In particular, "Determining the operator-machine assignment for machine interference problem and an empirical study in semiconductor test facility" by Chien, Zheng, and Lin, develops a methodology to determine the optimal assignment relationships between the test machines and the operators for different product mixes to enhance productivity and system performance, in which an empirical study has validates the proposed approach. "Therblig-based energy demand modeling methodology of machining process to support intelligent manufacturing" by Jia, Tang, and Lv, develops a mathematical model of energy demand of machining processes by linking the activity and energy demand units with machining state. Two cases including energy demand of turning process and energy demand of three grooving processes have shown its practical viability to estimate energy demand for the energy-oriented intelligent manufacturing. "Hierarchical indices to detect the tool condition change with high-dimensional equipment data for semiconductor manufacturing" by Yu, Lin, and Chien, investigates the ways to early detect the changes of equipment conditions for efficient equipment maintenance so that domain engineers can identify potential root causes by drilling down the proposed hierarchical indices. "Integrated data envelopment analysis and neural network model for forecasting performance of wafer fabrication operations" by Hsu constructs a model that integrates data envelopment analysis (DEA) and a back-propagation neural network (BPNN) for performance evaluation and forecast, in which an empirical study was conducted for a semiconductor company for validation.

The third cluster introduces novel approaches and applications for manufacturing intelligence in various domains. In particular, "An empirical study of design-of-experiment data mining for yield-loss diagnosis for semiconductor manufacturing" by Chien, Chang, and Wang, proposes a novel approach integrating Design-of-Experiment (DOE) and data mining to effectively extract "experimental designs" from big manufacturing data for narrowing the possible root causes that have been implemented as a function of engineering data analysis platform in the case company. "Tissue characterization of coronary plaque by kNN classifier with fractal-based features of IVUS RF-signal" by Uchino, Koga, Misawa, and Suetake, proposes a tissue characterization method for coronary plaques by using k-nearest neighbor $(\mathrm{kNN})$ method and fractal analysis-based feature that are collected from radiofrequency (RF) signals measured by the intravascular ultrasound (IVUS) method. "The construction of a hospital disease tracking and control system with diseases infection probability model" by Huang and Xie, addresses the application of Radio Frequency Identification (RFID) to monitor the infected patients and detect potential routes of disease transmission, in which a tree structure algorithm and an infection probability model were employed to trace the transmission routes, discover the correlations between carriers and suspected cases, and derive the infection probability. "GMVN oriented S-BOX knowledge expression and reasoning framework" by $\mathrm{Li}$, Xie, and Tang, proposes an intelligent integration framework of Semantic Bill of X (S-BOX) by combining with the technologies of ontology, Semantic Web Rule Language (SWRL), and Bill of $\mathrm{X}(\mathrm{BOX})$ to integrate the structures and operation rules into bill models and thus transform the strategy, decisions, management, and operations involved in Global Manufacturing Virtual Network (GMVN) into semantic reasoning activities.

The fourth cluster solves critical management problems involved in intelligent logistics and supply chains. "A multiobjective genetic algorithm for yard crane scheduling problem with multiple work lines" by Liang, Guo, Gen, and Jo, develops a multiobjective $0-1$ integer programming (MO01IP) model considering the minimum total completion time of all yard cranes and the maximization balanced distribution of the completion time at the same time to solve the yard scheduling problem. "An adaptive genetic algorithm for the time dependent inventory routing problem" by Cho, Lee, Lee, and Gen, proposes an adaptive genetic algorithm (AGA) to the time dependent inventory routing problem (TDIRP), in which inventory control and time dependent vehicle routing decisions for a set of retailers are made simultaneously over a specific planning horizon. "Optimization technique by 
genetic algorithms for international logistics" by Takeyasu and Kainosho, addresses a sea and air transportation problem by multi-step tournament selection with considering transportation cost and warehouse stock fees in the objective function.

While this special issue has successfully addressed some of critical research needs for manufacturing intelligence and innovations for digital manufacturing and operational excellence, we would like to express our sincere appreciations to all of the authors, the anonymous reviewers, and the Editor-in-Chief Professor Andrew Kusiak and the Assistant Babu Krishnamoorthy of Journal of Intelligent Manufacturing, for their invaluable contributions and great supports to this special issue.

\section{References}

Chen, L.-F., \& Chien, C.-F. (2011). Manufacturing intelligence for class prediction and rule generation to support human capital decisions for high-tech industries. Flexible Services and Manufacturing Journal, 23(3), 263-289.

Chien, C.-F., Chen, Y.-J., \& Peng, J.-T. (2010). Manufacturing intelligence for semiconductor demand forecast based on technology diffusion and product life cycle. International Journal of Production Economics, 128(2), 496-509.

Chien, C.-F., Hsu, C.-Y., \& Chen, P.-L. (2013a). Semiconductor fault detection and classification for yield enhancement and manufacturing intelligence. Flexible Services and Manufacturing Journal, 25(3), 367-388.

Chien, C.-F., Hsu, S.-C., \& Chen, Y.-J. (2013b). A system for online detection and classification of wafer bin map defect patterns for manufacturing intelligence. International Journal of Production Research, 51(8), 2324-2338.

Chien, C.-F., Hsu, C.-Y., \& Hsiao, C. (2011). Manufacturing intelligence to forecast and reduce semiconductor cycle time. Journal of Intelligent Manufacturing, 23(6), 2281-2294.

Chien, C.-F., Tseng, F., \& Chen, C. (2008). An evolutionary approach to rehabilitation patient scheduling: A case study. European Journal of Operational Research, 189(3), 1234-1253.
Chien, C.-F., Wang, H., \& Wang, M. (2007). A UNISON framework for analyzing alternative strategies of IC final testing for enhancing overall operational effectiveness. International Journal of Production Economics, 107(1), 20-30.

Chien, C.-F., Wu, C., \& Chiang, Y. (2012). Coordinated capacity migration and expansion planning for semiconductor manufacturing under demand uncertainties. International Journal of Production Economics, 135(2), 860-869.

Hsu, S., \& Chien, C.-F. (2007). Hybrid data mining approach for pattern extraction from wafer bin map to improve yield in semiconductor manufacturing. International Journal of Production Economics, 107(1), 88-103.

Hsu, C.-Y., Chien, C.-F., \& Chen, P. (2012). Manufacturing intelligence for early warning of key equipment excursion for advanced equipment control in semiconductor manufacturing. Journal of the Chinese Institute of Industrial Engineer, 29(5), 303-313.

Kuo, C.-J., Chien, C.-F., \& Chen, C.-D. (2011). Manufacturing intelligence to exploit the value of production and tool data to reduce cycle time. IEEE Transactions on Automation Science and Engineering, 8(1), 103-111.

Leachman, R., Ding, S., \& Chien, C.-F. (2007). Economic efficiency analysis of wafer fabrication. IEEE Transactions on Automation Science and Engineering, 4(4), 501-512.

Lin, K., \& Chien, C.-F. (2009). Cluster analysis of genome-wide expression data for feature extraction. Expert Systems with Applications, 36(2), 3327-3335.

Liu, C.-W., \& Chien, C.-F. (2013). An intelligent system for wafer bin map defect diagnosis: An empirical study for semiconductor manufacturing. Engineering Applications of Artificial Intelligence, 26(5-6), 1479-1486.

Wu, J.-Z., Chien, C.-F., \& Gen, M. (2012a). Coordinating strategic outsourcing decisions for semiconductor assembly using a bi-objective genetic algorithm. International Journal of Production Research, $50(1), 235-260$.

Wu, J.-Z., Hao, X.-C., Chien, C.-F., \& Gen, M. (2012b). A novel bivector encoding genetic algorithm for the simultaneous multiple resources scheduling problem. Journal of Intelligent Manufacturing, 23(6), 2255-2270. 